\title{
Pair condensation of bosonic atoms induced by optical lattices
}

\author{
María Eckholt ${ }^{1}$ and Juan José García-Ripoll ${ }^{2}$ \\ ${ }^{1}$ Max-Planck-Institut für Quantenoptik, Hans-Kopfermann-Str. 1, Garching, D-85478, Germany. \\ ${ }^{2}$ Facultad de CC. Físicas, Universidad Complutense de Madrid, \\ Ciudad Universitaria s/n, Madrid, E-28040, Spain.
}

\begin{abstract}
We design a model of correlated hopping for bosonic atoms in optical lattices. Such model exhibits three kinds of phases, comprehending a Mott insulator, a charge density wave and a pair quasi-condensate. The implementation of the model relies on two-state atoms embedded in statedependent lattices and having spin-dependent interactions. Contrary to other models of pairing, our design is not based on perturbative effects and should be observable in current experiments.
\end{abstract}

Since the achievement of Bose-Einstein condensation in alkali atoms [1, 2, 3], we have witnessesed two major breakthroughs in the many-body physics of cold atoms. One is the realization of Cooper pairing and the BCS to BEC transition with fermions [4, 5, 6]. The other one is the implementation of lattice Hamiltonians using neutral atoms in optical lattices [7, 8]. Supported by this success, many theoretical papers suggest using cold atoms with two goals: the quantum simulation of well known Hamiltonians such as Hubbard models [8] and spin lattices [9], and the quest for new physics such as bosonic quantum Hall effect [10, 11] and lattice gauge theories [12, 13]. In this work we aim at the latter, introducing a robust mechanism of pairing that works for bosonic atoms, opens theoretical challenges and is suited for the recent experiments in optical superlattices 14, 15].

Pairing is a central concept in strongly correlated states. In particular, it is the essence of ordinary BCS superconductivity. In BCS theory, an attractive interaction mediated by a phonon bath is the basis by which electrons merge into bosonic pairs that conduct electricity without friction. Similar phenomena appear in the BCS pairing of fermionic atoms, where the natural attraction is enhanced by Feschbach resonances [4, 5, 6].

Another, less known mechanism for pairing is correlated hopping. It appears naturally in fermionic tightbinding models 16, 17, 18, 19, 20, 21, 22 and in quantum magnetism [23], consisting on the motion of particles being influenced by the environment. This is normally reflected by terms of the form $n_{i} a_{j}^{\dagger} a_{k}$ appearing in the Hamiltonian. Correlated hopping could lead to the formation of bound electron pairs [19, 20] and it has been put forward as an explanation for high $T_{c}$ superconductivity [24, 25].

We will introduce a different mechanism for pairing, which is based on transport-inducing collisions. As shown in Fig. 1, when atoms collide they can mutate their internal state. If the atoms are placed in a statedependent optical lattice, whenever such a collision happens, the pair of atoms must jump to a different site associated to their new state. For deep enough lattices, as in the Mott insulator experiments 7], this coordinated jump of pairs of particles will be the dominant process and the atoms will become a superfluid of pairs.

This work consists of three parts. The first one begins with a setup of cold atoms in a deep optical superlattice. We show how collisions combined with state-dependent lattices lead to a strong correlated tunnelling, as described above. The outcome is an effective Hamiltonian which can be related to the experimental parameters. We will then draw a realistic phase diagram using both Gutzwiller [26] and Matrix Product States (MPS) 27] variational methods. Both procedures essentially identify three kinds of phases: a charge-density wave (CDW), a Mott insulator (MI) and a pair condensate or pair superfluid (PSF). In the last part of the paper we consider different experimental procedures to detect the different insulating and superfluid orders.

Let us begin by considering the setup in Fig. 11, where

a)

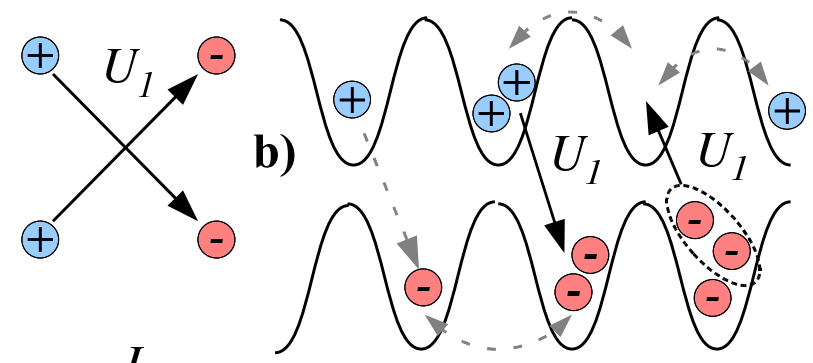

c)

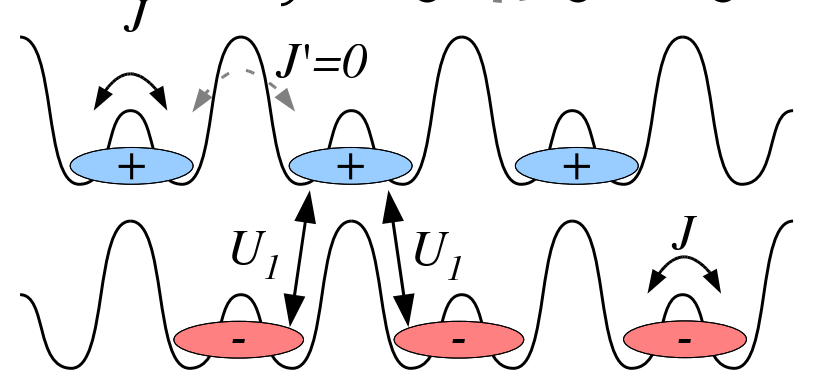

FIG. 1: (a) Two atoms in state $|+\rangle$ collide and change into state $|-\rangle$. (b) When trapped in a state-dependent optical lattice, changing the state implies also jumping between lattice sites. We plot some forbidden (dashed) and allowed (solid) processes. Only pairs of atoms can hop between lattices. (c) In order to maximize the overlap between atoms in different states and thus the strength of correlated hopping, in this paper we consider two state-dependent superlattices. 
bosonic atoms in two internal states, $a_{+}$and $a_{-}$, are trapped in state-dependent superlattices that spatially overlap. The hopping outside a double well is negligible. In this limit, we have two bands of localized states with energies proportional to the hopping amplitude on a double well, $+J$ and $-J$. In particular the lowest band will be given by the bosonic states $c_{2 k}:=\frac{1}{\sqrt{2}}\left(a_{2 k+}+a_{2 k+1+}\right)$, and $c_{2 k+1}:=\frac{1}{\sqrt{2}}\left(a_{2 k+1-}+a_{2 k+2-}\right)$.

If the interaction is weak compared to the band gap $2 J$, only the coupling between states in the same band will be relevant. We will focus on a particular situation in which the trapped states are actually dressed states, $a_{ \pm}:=\left(a_{\uparrow} \pm a_{\downarrow}\right) / \sqrt{2}$, and the interaction is diagonal in those other operators, $a_{\uparrow}$ and $a_{\downarrow}$. This situation is reached by combining the optical lattice configuration in Ref. [28] with asymmetric on-site interactions

$$
H_{k}=\frac{U_{0}}{2}:\left(n_{\uparrow}+n_{\downarrow}\right)^{2}:+U_{1} n_{\uparrow} n_{\downarrow} .
$$

Note that the term $U_{1} n_{\uparrow} n_{\downarrow}$ is not invariant under rotations of the internal state of the atoms. When expressed in the $| \pm\rangle$ basis, it is this term that scatters pairs of atoms from one cloud to another [Fig. 13]. Since the superlattices have a relative displacement, these collisions give rise to transport. Using the approximation $U_{0}, U_{1} \ll 2 J$ one arrives at our central model

$$
H=\sum_{\langle i, j\rangle}\left[U:\left(n_{i}+n_{j}\right)^{2}:+V n_{i} n_{j}-t c_{i}^{\dagger 2} c_{j}^{2}\right]
$$

where $U=\left(2 U_{0}+U_{1}\right) / 16, V=-U_{1} / 8$, and $t=U_{1} / 16$.

Out of these terms, $V$ favors neighboring sites to be filled, $t$ is the correlated hopping that spreads pairs of atoms through the lattice, and $U \geq 0$ restricts the total number of atoms preventing collapse. If $U=0$ and $V=$ 0 , we expect the bosons to form a superfluid of paired particles, while for strong interactions there should be a quantum phase transition to an insulator.

The first evidence of pairing is obtained by studying a lattice with only two particles. This setup has two potential ground states: one with both particles isolated and zero energy, and a delocalized state of a single pair $\left|\psi_{\text {pair }}\right\rangle \propto \sum_{i} c_{i}^{\dagger 2}|0\rangle$ with energy $E_{2}=2(U-V)$. Therefore, if $U<V$ or $U_{0}<U_{1} / 2$, the pair of bosons will be bound, $E_{2}<0$. An important question is whether this criterion survives in the presence of other atoms, or if many-body effects may destroy the pairing.

In order to study the many-body physics of our model we have used the Gutzwiller ansatz, which is a variational method based on the product state [26]

$$
\left|\psi_{\mathrm{GW}}\right\rangle=\prod_{i} \sum_{n} \frac{1}{\sqrt{n !}} f_{n} c_{i}^{\dagger n}|0\rangle
$$

Minimizing the expectation value of the free energy, $F:=H-\mu N$, with respect to the variables $f_{n}$ under the constrain of fixed norm, $\sum\left|f_{n}\right|^{2}=1$, one obtains the phase diagram in the phase space of interaction and chemical potential, $\left(U_{1}, U_{0}, \mu\right)$. For the sake of simplicity we have used $U_{1}:=1$ as unit of energy everywhere.

The results are shown in Fig. 2, where we plot the expectation values of the density, $\bar{n}=\langle n\rangle=\left\langle c^{\dagger} c\right\rangle$, the particle number variance, $\Delta n:=\sqrt{\left\langle n^{2}-\bar{n}^{2}\right\rangle}$, and what we identify as the order parameter of the paired superfluid, $\left\langle c^{2}\right\rangle$. From the zeros of the density fluctuations we recognize the Mott regions with $\bar{n}=1,2$ and 3 particles per site. The boundary of the region with $\bar{n}=1$ can be estimated analytically using a Gutzwiller state with nonzero components $f_{0}, f_{1}$ and $f_{2}$, which gives

$$
\mu_{p}=\frac{U_{0}}{2}, \text { and } \mu_{h}=U_{0}+\frac{1}{4} .
$$

These are the energies to add a particle or to make a hole in this insulating lobe. If $\mu<\mu_{h}$, the Gutzwiller ansatz gives fractional densities but, as we will see below, this is an artifact of the uniform trial wavefunction. If $\mu>\mu_{p}$ and $U_{0}<U_{1} / 2$, we obtain a region of nonzero $\left\langle c^{2}\right\rangle$. We take this as a sign of a long-range coherence in the two-body density matrix which cannot be attributed to an ordinary Bose-Einstein condensate, since the same simulation gives $\langle c\rangle=0$

The Gutzwiller ansatz does not reproduce accurately neither the location of phase transitions nor the behavior of correlators. For instance, $C_{\Delta}^{1}:=\left\langle c_{i}^{\dagger} c_{i+\Delta}\right\rangle$ and $C_{\Delta}^{2}:=\left\langle c_{i}^{\dagger 2} c_{i+\Delta}^{2}\right\rangle$ computed with Eq. (3) are both uniform functions. In order to study these properties, we have used the MPS method [27] to estimate variationally the ground state of our model. The MPS is a more complex wavefunction that, for large enough computational resources and not too large systems, should reproduce the correlations in the superfluid regime. Calculations with up to 40 sites reveal that matrices of size $D=40$ are enough to pinpoint the different phases.

In Fig. 3 we plot the same quantities as for the Gutzwiller ansatz. The contour plots reveal that the $\bar{n}=1$ and $\bar{n}=2$ insulating regions still exist, with a similar size and shape as in the Gutzwiller case. As we explain later, the region above $\mu_{p}$ again shows a paired superfluid behavior, which extends even beyond the limit $U_{0}=U_{1} / 2$ where the binding energy of an isolated pair becomes positive [Fig. [3] ]. The biggest difference is in the triangle $0 \leq \mu<\mu_{h}$. In this region of low density, the bosons arrange forming a charge density wave, i. e. a pattern alternating 0 and 1 atom per site.

Regarding the superfluid, quasi-long-range order is identified by a slow decay of the off-diagonal elements of the single- and two-particle density matrix. As shown in Fig. 4, the single particle correlator $C_{\Delta}^{1}=\bar{\delta}_{\Delta, 0}$ is only different from zero at $\Delta=0$, where it becomes the density. This could have indicated the presence of a Mott phase, were it not for the nonzero value of $\Delta n$ (Fig. $3 \mathrm{~b}$ ) and of the two particle correlator, $C_{\Delta}^{2}$, that decays slowly 
at long distances. Due to the size of our simulations, we have not yet been able to determine the behavior of $C_{\Delta}^{2}$, but numerical fits of curves like Fig. 4 suggest an algebraic decay, $C_{\Delta}^{2} \sim \Delta^{-\alpha}$, with a nonuniversal exponent around $\alpha<0.7$ that depends on $U_{0}$ and $\mu$.

Let us now introduce another possible experimental setup, illustrated in Fig. 1 b and where two lattices trapping the two different species are shifted by half a period or $\lambda / 4$. As usual, the trapped states and the interaction are described in different basis. One can expand the interacting Hamiltonian in terms of Wannier functions together with operators defining the trapped states. This leads to Eq. (2) with: $U=\left(2 U_{0}+U_{1}\right) / 4$, $V=-\left(2 \beta U_{0}+U_{1}\right) / 2$ and $t=-U_{1} / 4$. Here, $U_{0}$ and $U_{1}$ are the on-site interactions in the atomic basis and the factor $\beta=\int|w(x)|^{2}|w(x-\lambda / 4)|^{2} d x / \int|w(x)|^{4} d x \ll 1$, computed using the Wannier functions $w(x)$, measures the relative strength of interaction between different lattices with respect to those on the same lattice site.

The phases that appear in our setup can all be recognized experimentally. First of all, the MI and the CDW have both a well defined number of particles per site and no coherence. Their time of flight pictures will have no interference fringes [7] and the noise correlation will exhibit peaks at certain momenta [29, 30]. In addition, the CDW corresponds to a setup where either $n_{+}$or $n_{-}$are uniformly zero. Finally, the energy gap in these insulators can be probed by static [7] or spectroscopic means 31 .

Regarding the pair superfluid, it is a perfect "conductor" with a gapless excitation spectrum. Lacking singleparticle order, $C_{\Delta}^{1} \sim 0$ a.e., it will also not show interference patterns in the time of flight images. In order to measure $C_{\Delta}^{2}$ and detect the pairing, we suggest to use photoassociation and to build molecules out of pairs of atoms. Since the molecules will be built on-site, the nonzero correlator $C_{\Delta}^{2}$ will translate into long-range order for the molecules, which should now exhibit an interference pattern in time-of-flight images, slightly blurred due to the phase fluctuations inherent to $1 \mathrm{D}$.

Finally, all energy scales are referenced to the asymmetry of the interactions, $U_{1}$. Therefore, the pair binding energy can be potentially as strong as the strength of the on-site interaction in a typical Mott insulator [7], about $1 \mathrm{kHz}$. In practice, while the most commonly used atoms such as Rubidium have smaller asymmetries, they can be enhanced using Feschbach resonances.

Summing up, we have introduced a mechanism by which bosonic atoms with repulsive interactions can exhibit correlated hopping and pairing. The model (2) exhibits multiple phases, among which the most relevant is a superfluid of paired bosons. All phases are connected by second order quantum phase transitions and can be produced and identified using variations of current experiments [14, 15]. These ideas can be generalized to higher dimensions and other other kinds of interaction.
We thank Miguel Angel Martín-Delgado for useful discussions. M.E. acknowledges support from the CONQUEST project. J.J.G.R acknowledges financial support from the Ramon y Cajal Program of the Spanish M.E.C., from U.S. NSF Grant No. PHY05-51164 and from the spanish projects FIS2006-04885 and CAM-UCM/910758.

[1] M. H. Anderson, J. R. Ensher, M. R. Matthews, C. E. Wieman, and E. A. Cornell, Science 269, 198 (1995).

[2] K. B. Davis, M.-O. Mewes, M. R. Andrews, N. J. van Druten, D. S. Durfee, D. M. Kurn, and W. Ketterle, Phys. Rev. Lett. 75, 3969 (1995).

[3] C. C. Bradley, C. A. Sackett, J. J. Tollett, and R. G. Hulet, Phys. Rev. Lett. 75, 1687 (1995).

[4] C. A. Regal, M. Greiner, and D. S. Jin, Phys. Rev. Lett. 92, 040403 (2004).

[5] M. W. Zwierlein, C. A. Stan, C. H. Schunck, S. M. Raupach, A. J. Kerman, and W. Ketterle, Phys. Rev. Lett. 92, 120403 (2004).

[6] T. Bourdel, L. Khaykovich, J. Cubizolles, J. Zhang, F. Chevy, M. Teichmann, L. Tarruell, S. J. Kokkelmans, and C. Salomon, Phys. Rev. Lett. 93, 050401 (2004).

[7] M. Greiner, O. Mandel, T. Esslinger, T. W. Hänsch, and I. Bloch, Nature 415, 39 (2002).

[8] D. Jaksch, C. Bruder, J. I. Cirac, C. W. Gardiner, and P. Zoller, Phys. Rev. Lett. 81, 3108 (1998).

[9] L.-M. Duan, E. Demler, and M. D. Lukin, Phys. Rev. Lett. 91, 090402 (2003).

[10] N. K. Wilkin and J. M. F. Gunn, Phys. Rev. Lett. 84, 6 (2000).

[11] B. Paredes, P. Fedichev, J. I. Cirac, and P. Zoller, Phys. Rev. Lett. 87, 010402 (2001).

[12] D. Jaksch and P. Zoller, New J. Phys. 5, 56 (2003).

[13] K. Osterloh, M. Baig, L. Santos, P. Zoller, and M. Lewenstein, Phys. Rev. Lett. 95, 010403 (2005).

[14] M. Anderlini, P. J. Lee, B. L. Brown, J. Sebby-Strabley, W. D. Phillips, and J. V. Porto, Nature 448, 452 (2007).

[15] S. Fölling, S. Trotzky, P. Cheinet, M. Feld, R. Saers, A. Widera, T. Müller, and I. Bloch, Nature 448, 1029 (2007).

[16] M. E. Foglio and L. M. Falicov, Phys. Rev. B 20, 4554 (1979).

[17] I. N. Karnaukhov, Phys. Rev. Lett. 74, 5285 (1995).

[18] I. N. Karnaukhov, Phys. Rev. Lett. 73, 1130 (1994).

[19] L. Arrachea, A. A. Aligia, E. Gagliano, K. Hallberg, and C. Balseiro, Phys. Rev. B 50, 16044 (1994).

[20] L. Arrachea and A. A. Aligia, Phys. Rev. Lett. 73, 2240 (1994).

[21] J. de Boer, V. E. Korepin, and A. Schadschneider, Phys. Rev. Lett. 74, 789 (1995).

[22] J. Vidal and B. Douçot, Phys. Rev. B 65, 045102 (2001).

[23] K. P. Schmidt, J. Dorier, A. Lauchli, and F. Mila, Phys. Rev. B 74, 174508 (pages 7) (2006).

[24] J. E. Hirsch, Physica C 158, 326 (1989).

[25] F. Marsiglio and J. E. Hirsch, Phys. Rev. B 41, 6435 (1990).

[26] W. Krauth, M. Caffarel, and J.-P. Bouchaud, Phys. Rev. B 45, 3137 (1992).

[27] F. Verstraete, D. Porras, and J. I. Cirac, Phys. Rev. Lett. 93, 227205 (2004). 
[28] J. J. García-Ripoll and J. K. Pachos, New J. Phys. 9, 139 (2007).

[29] E. Altman, E. Demler, and M. D. Lukin, Phys. Rev. A 70, 013603 (2004).

[30] S. Fölling, F. Gerbier, A. Widera, O. Mandel, T. Gericke, and I. Bloch, Nature 434, 481 (2005).

[31] T. Stöferle, H. Moritz, C. Schori, M. Köhl, and T. Esslinger, Phys. Rev. Lett. 92, 130403 (2004). 

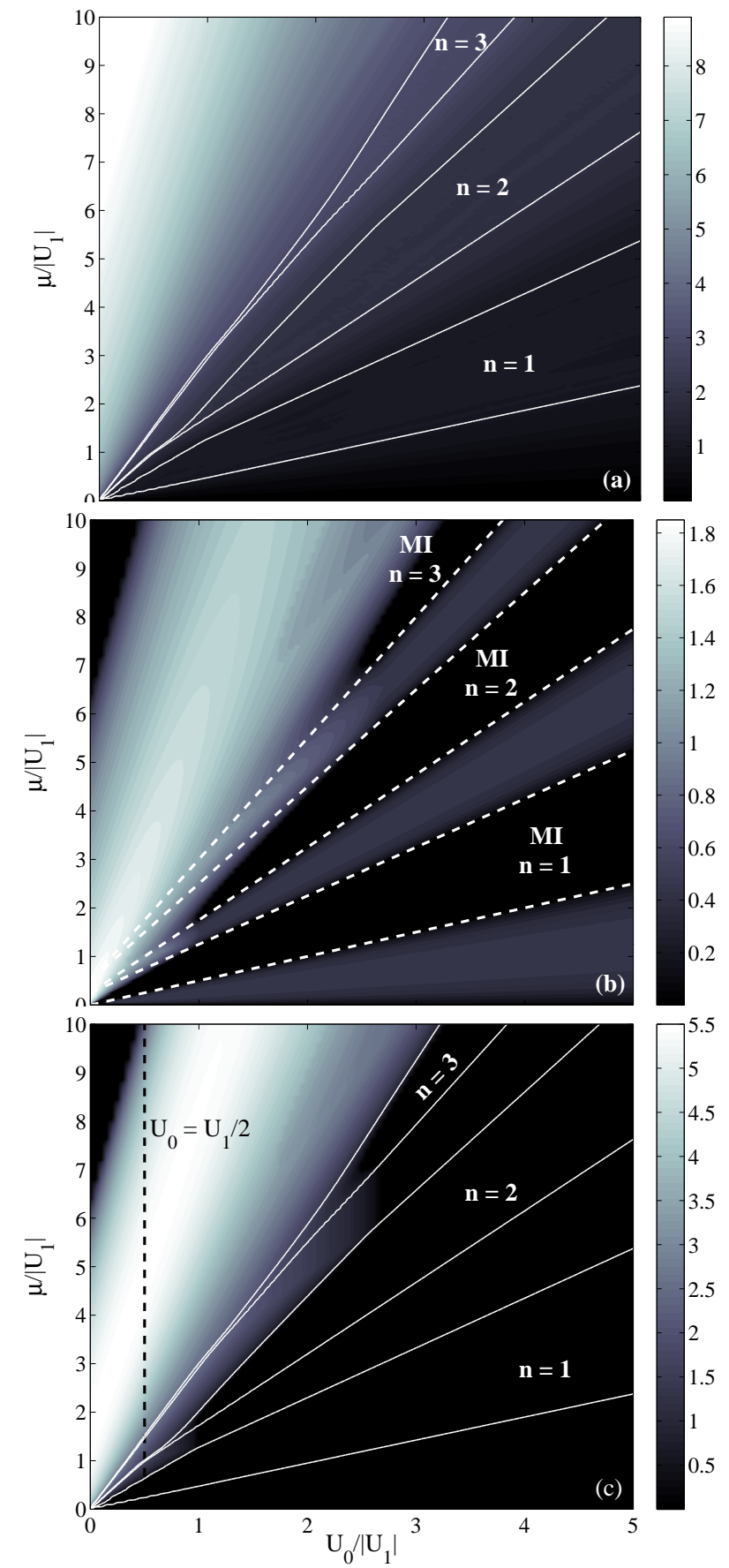

FIG. 2: Ground state properties estimated with the Gutzwiller wavefunction (3). Grayscale plots of (a) the density, $\bar{n}$, (b) density fluctuations, $\Delta n$, and (c) pair condensate order parameter $\left\langle c^{2}\right\rangle$. Dashed lines mark the analytical estimates (4), while solid lines delimit regions of integer filling. All plots cover the same region $\left(U_{0}, \mu\right) \in\left[0,5 U_{1}\right] \times\left[0,10 U_{1}\right]$. 

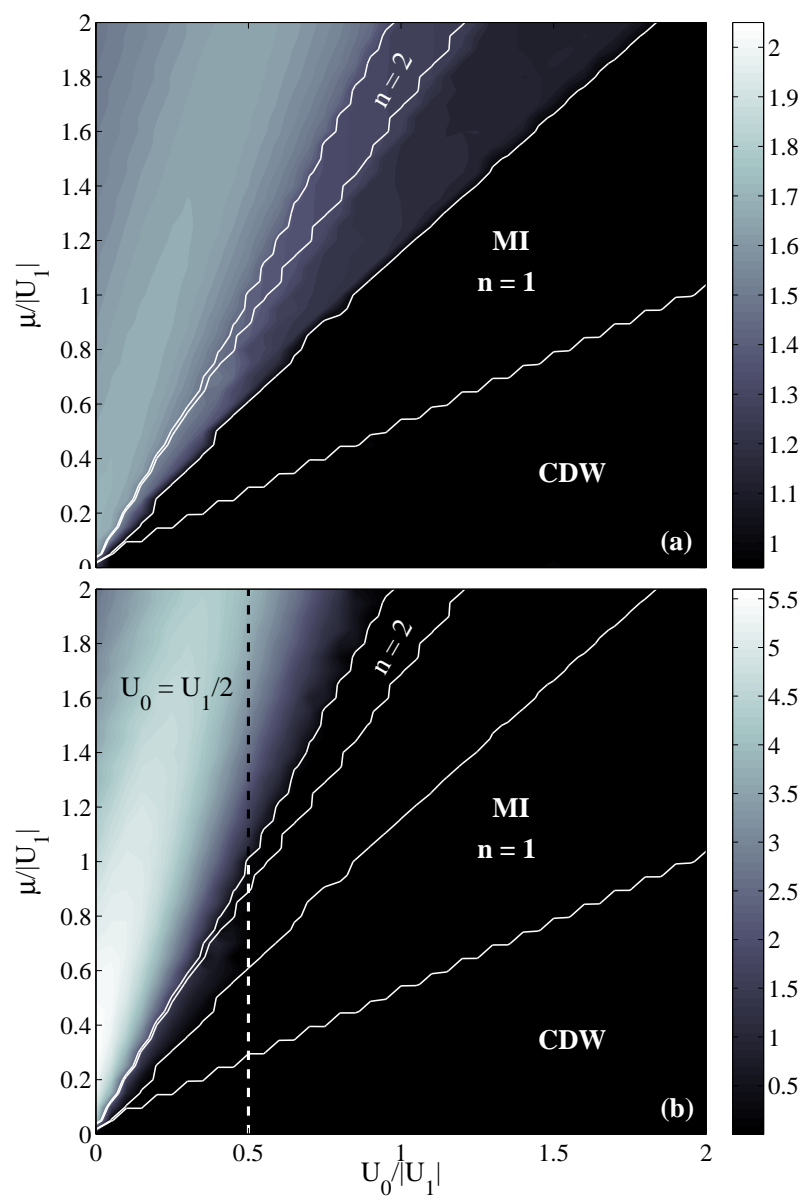

FIG. 3: Ground state properties estimated with the MPS method. We plot (a) the fluctuations of the density, $\Delta n$, and (b) the averaged two-particle correlator, $\sum_{\Delta}\left|C_{\Delta}^{2}\right| / L$, where $C_{\Delta}^{2}:=\left\langle c_{i}^{\dagger 2} c_{i+\Delta}^{2}\right\rangle$. Solid lines delimit regions of integer filling. All plots cover the same region $\left(U_{0}, \mu\right) \in\left[0,2 U_{1}\right] \times\left[0,2 U_{1}\right]$.

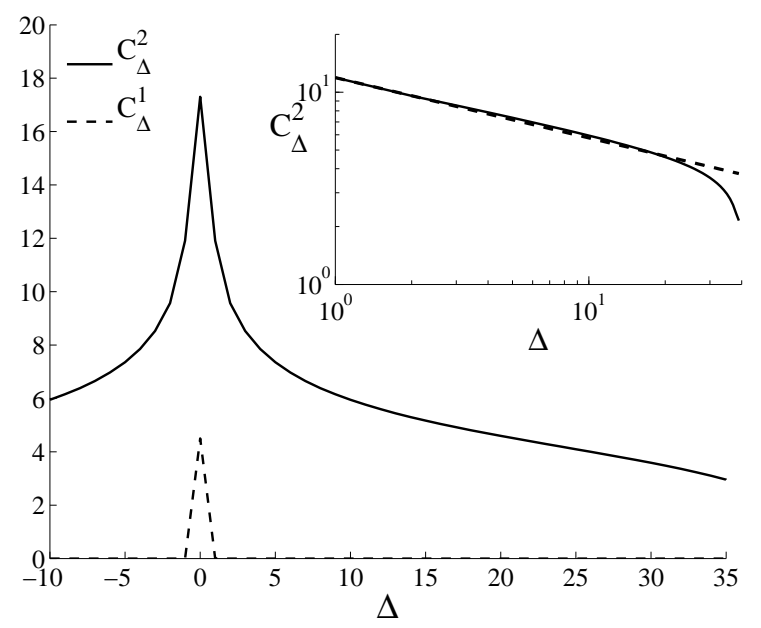

FIG. 4: Single particle, $C_{\Delta}^{1}:=\left\langle c_{i}^{\dagger} c_{i+\Delta}\right\rangle$ (dashed), and two particle, $C_{\Delta}^{2}:=\left\langle a_{i}^{\dagger 2} a_{i+\Delta}^{2}\right\rangle$, correlator (solid) for $\mu / U_{1}=$ $0.9, U_{0} / U_{1}=0$ and a lattice with 40 sites. The inset repeats the plot for $C_{\Delta}^{2}$ in log-log scale, with a fit $C_{\Delta}^{2} \propto \Delta^{0.32}$ (dash). 